\title{
Stable accretion from a cold disc in highly magnetized neutron stars
}

\author{
S. S. Tsygankov ${ }^{1,2}$, A. A. Mushtukov ${ }^{3,2}$, V. F. Suleimanov ${ }^{4,2}$, V. Doroshenko ${ }^{4}$, P. K. Abolmasov ${ }^{1}$, \\ A. A. Lutovinov ${ }^{2,5}$, and J. Poutanen ${ }^{1,6,7}$ \\ 1 Tuorla Observatory, Department of Physics and Astronomy, University of Turku, Väisäläntie 20, 21500 Piikkiö, Finland \\ e-mail: sergey.tsygankov@utu.fi \\ 2 Space Research Institute of the Russian Academy of Sciences, Profsoyuznaya Str. 84/32, Moscow 117997, Russia \\ 3 Anton Pannekoek Institute, University of Amsterdam, Science Park 904, 1098 XH Amsterdam, The Netherlands \\ ${ }^{4}$ Institut für Astronomie und Astrophysik, Universität Tübingen, Sand 1, 72076 Tübingen, Germany \\ 5 Moscow Institute of Physics and Technology, Moscow region, Dolgoprudnyi 141700, Russia \\ 6 Nordita, KTH Royal Institute of Technology and Stockholm University, Roslagstullsbacken 23, 10691 Stockholm, Sweden \\ 7 Kavli Institute for Theoretical Physics, University of California, Santa Barbara, CA 93106, USA
}

Received 14 December 2016 / Accepted 21 September 2017

\begin{abstract}
Aims. The aim of this paper is to investigate the transition of a strongly magnetized neutron star into the accretion regime with very low accretion rate.

Methods. For this purpose, we monitored the Be-transient X-ray pulsar GRO J1008-57 throughout a full orbital cycle. The current observational campaign was performed with the Swift/XRT telescope in the soft X-ray band (0.5-10 keV) between two subsequent Type I outbursts in January and September 2016.

Results. The expected transition to the propeller regime was not observed. However, transitions between different regimes of accretion were detected. In particular, after an outburst, the source entered a stable accretion state characterised by an accretion rate of $\sim 10^{14}-10^{15} \mathrm{~g} \mathrm{~s}^{-1}$. We associate this state with accretion from a cold (low-ionised) disc of temperature below $\sim 6500 \mathrm{~K}$. We argue that a transition to this accretion regime should be observed in all X-ray pulsars that have a certain combination of the rotation frequency and magnetic field strength. The proposed model of accretion from a cold disc is able to explain several puzzling observational properties of X-ray pulsars.
\end{abstract}

Key words. accretion, accretion disks - magnetic fields - X-rays: binaries - X-rays: individuals: GRO J1008-57

\section{Introduction}

The properties of an accretion disc strongly depend on the parameters of the compact object and the binary system as a whole. At the same time, they also determine the observational appearance of an accreting object. As a consequence, some of the physical phenomena typical for one class of objects may not be observable in another. For instance, transitions between different ionisation states of an accretion disc are commonly considered to be responsible for bright outbursts in dwarf novae and soft X-ray transients (see, e.g. reviews by Cherepashchuk 2000; Lasota 2001; Warner 2003), but previously were not considered for X-ray pulsars (XRPs). The transition is associated with the so-called thermal-viscous instability that arises as a result of the partial ionisation of hydrogen in an accretion disc (Meyer \& Meyer-Hofmeister 1984; Smak 1984; Mineshige et al. 1993; Cheng et al. 1992; Chen et al. 1997).

The ionisation state of the plasma in the disc determines the disc opacity and the equation of state, and, therefore, the viscosity (see, e.g. the review by Lasota 2001). In a cold disc that consists mainly of atomic hydrogen, the viscosity and thus the accretion rate are low, so that the accreted matter accumulates in the disc. In the hot state, the hydrogen is mainly ionised and the disc viscosity dramatically increases, which allows most of the accumulated matter to be rapidly accreted onto a compact object. The critical effective temperature defining the two states is the hydrogen ionisation temperature, which is $\sim 6500 \mathrm{~K}$ (see Sect. 3.5 in Warner 2003). The transition between the two states is accompanied by a heating wave originating either from the inner or outer part of the accretion disc (Smak 1984). The accretion process becomes stable again when the temperature falls for some reason below the critical value across the entire disc, that is, in the case of a low mass-accretion rate (Lasota 1997),

$\dot{M}<\dot{M}_{\text {cold }} \simeq 3.5 \times 10^{15} r_{10}^{2.65} M_{1.4}^{-0.88} \mathrm{~g} \mathrm{~s}^{-1}$,

where $r_{10}$ is the inner disc radius ${ }^{1}$ and $M_{1.4}$ is the neutron star mass in units of $1.4 M_{\odot}$.

From another perspective, at very low mass accretion rates onto a magnetized neutron star, the so-called "propeller effect" may occur as an abrupt luminosity drop below some critical value (Illarionov \& Sunyaev 1975). This rapid cessation of accretion is caused by a centrifugal barrier produced by the rotating magnetosphere if it moves faster than the local Keplerian velocity. In other words, when the magnetospheric radius

$R_{\mathrm{m}} \simeq 2.5 \times 10^{8} k M_{1.4}^{1 / 7} R_{6}^{10 / 7} B_{12}^{4 / 7} L_{37}^{-2 / 7} \mathrm{~cm}$

1 We define $Q_{x}=Q / 10^{x}$ in cgs units if not stated otherwise. 
(the neutron star magnetic momentum is taken to be $\mu=B R^{3} / 2$ ) is larger than the corotation radius,

$R_{\mathrm{c}}=\left(\frac{G M P^{2}}{4 \pi^{2}}\right)^{1 / 3} \simeq 1.68 \times 10^{8} M_{1.4}^{1 / 3} P^{2 / 3} \mathrm{~cm}$.

The limiting luminosity for the onset of the propeller can be estimated by equating the corotation and magnetospheric radii (see, e.g. Tsygankov et al. 2016a):

$L_{\text {prop }} \simeq \frac{G M \dot{M}_{\text {prop }}}{R} \simeq 4 \times 10^{37} k^{7 / 2} B_{12}^{2} P^{-7 / 3} M_{1.4}^{-2 / 3} R_{6}^{5} \mathrm{erg} \mathrm{s}^{-1}$,

where $P$ is the neutron star rotational period in seconds and $\dot{M}$ is the mass-accretion rate. Factor $k$ accounts for the details of interaction of the accretion flow with the magnetosphere, relating its size to the Alfvén radius, that is, $k=R_{\mathrm{m}} / R_{\mathrm{A}}$. In the case of disc accretion, it is usually assumed to be $k=0.5$ (Ghosh \& Lamb 1978).

In the case of rapidly rotating accreting XRPs (using $4 \mathrm{U} 0115+63$ and $\mathrm{V} 0332+53$ as case studies), Tsygankov et al. (2016a) showed that an observation of the transition of the accretion disc to the cold state is impossible because of the onset of the propeller regime at much higher mass accretion rates than the rate required for the accretion disc to have a temperature below $6500 \mathrm{~K}$ at the inner radius. At the same time, the overall shape and energetics of the outburst were shown to correspond to expectations from the disc instability model.

XRPs with spin periods that are sufficiently long to reduce the centrifugal barrier are required to reach a sufficiently low mass-accretion rate to switch the accretion disc to the cold state before the transition to the propeller regime. A few XRPs with long spin periods exist, one of which, GRO J1008-57, shows very predictable Type I outbursts every periastron passage with long quiescence periods between the outbursts (Kühnel et al. 2013). This source was selected for the monitoring campaign that covered a full orbital cycle and aimed to study the source properties at a very low mass-accretion rate.

GRO J1008-57 was discovered as an XRP with a period of $93.587 \pm 0.005 \mathrm{~s}$ by the BATSE instrument on board the Compton Gamma-Ray Observatory (CGRO) during the bright outburst in 1993 (Stollberg et al. 1993). Its transient nature was associated with the Be type of the optical companion (B1-B2 Ve star; Coe et al. 2007). GRO J1008-57 shows giant, Type II, and Type I (associated with the periastron passage) outbursts. The distance to the source was estimated to be $5.8 \mathrm{kpc}$ (Riquelme et al. 2012). The orbital parameters of the binary system were determined using the data from several observatories: the orbital period $P_{\text {orb }}=249.46 \pm 0.10 \mathrm{~d}$, the projected semimajor axis $a_{\mathrm{x}} \sin i=530 \pm 60 \mathrm{lt}-\mathrm{s}$, the longitude of periastron $\omega=-26 \pm 8 \mathrm{deg}$, and the eccentricity $e=0.68 \pm 0.02$ (Levine \& Corbet 2006; Coe et al. 2007; Kuehnel et al. 2012). The presence of a cyclotron line at $\sim 88 \mathrm{keV}$ in the spectrum of the source was first suggested based on CGRO/OSSE data (Shrader et al. 1999) and was later confirmed with Suzaku at $E_{\text {cyc }}=75.5 \mathrm{keV}$ (Yamamoto et al. 2013). This allows us to estimate a magnetic field of the neutron star of $\sim 8 \times 10^{12} \mathrm{G}$.

In this work we analyse the data obtained with the Swift/XRT telescope during one full orbital cycle of Be/XRP GRO J1008-57 between the two consequent Type I outbursts in January and September 2016. The unique combination of high sensitivity and flexibility of scheduling for the Swift observatory allowed us to perform a detailed long-term observational campaign aimed at reaching accretion regimes that have never been investigated before. Knowledge of the magnetic field strength of the neutron star as well as of other parameters of the system allowed us to interpret the observed temporal behaviour in terms of the accretion disc instability and to construct the model of accretion from a cold disc in highly magnetized neutron stars.

\section{Data analysis and results}

\subsection{Swift/XRT observations}

The Swift observatory (Gehrels et al. 2004) provides the possibility of performing long-term observation campaigns of faint X-ray sources using the onboard focusing XRT telescope (Burrows et al. 2005). This work is based on XRT data collected between two consequent Type I outbursts in January and September 2016 (Kretschmar et al. 2016; Nakajima et al. 2016). Observations were performed every 3-4 days in photon-counting (PC) mode. The spectrum in each observation was extracted using the online tools ${ }^{2}$ provided by the UK Swift Science Data Centre (Evans et al. 2009).

Spectra were fitted with the XSPEC package using the Cash statistic (Cash 1979) after they were grouped to have at least one count per bin. To avoid any problems caused by the calibration uncertainties at low energies ${ }^{3}$, we restricted our spectral analysis to the $0.7-10 \mathrm{keV}$ band.

To estimate the bolometric correction factor, we used the results obtained by Kühnel et al. (2013), who demonstrated the dependence of the source spectrum on its luminosity. Using the spectral parameters in the faint state of GRO J1008-57, we obtained an estimate for the bolometric correction factor $K_{\text {bol }} \sim 1.6$. We note that the remaining uncertainty in the correction factor does not influence any of the conclusions. In the following analysis, we apply this correction to the Swift/XRT data and refer to the bolometric fluxes and luminosities, unless stated otherwise.

\subsection{Timing analysis}

The light curve of GRO J1008-57 obtained from the Swift/XRT data is shown in Fig. 1. Originally, the observations were requested in order to detect the transition of GRO J1008-57 to the propeller regime (see Sect. 3) at the limiting luminosity of $L_{\text {prop }}=5.6 \times 10^{33} \mathrm{erg} \mathrm{s}^{-1}$ (shown in the upper panel of Fig. 1 with the horizontal dashed line). However, at some point in the declining phase of the outburst, the source unexpectedly stopped fading around MJD 57440 at a luminosity of around $10^{35} \mathrm{erg} \mathrm{s}^{-1}$ (shown in the same figure with the dotted line), which is one and a half orders of magnitude higher than $L_{\text {prop. During the next }}$ seven months, the source continued to fade, but at a much lower rate characterised by an e-folding time of $\tau \sim 145$ days in contrast to $\tau \sim 5.5$ days before the transition. Strictly speaking, the flux decline during the low-level state is better fitted with a power-law of index $\sim-0.7$.

Eventually, the monitoring covered the whole orbital cycle, with the sharp brightening of the source on MJD 57648 signifying the next Type I outburst during the subsequent periastron passage (Nakajima et al. 2016). The transition of the source to the propeller state has therefore not been observed.

Despite the short duration of individual Swift observations and the long spin period of the source, the pulsations are easily detectable during the outburst. Similarly to what has been

\footnotetext{
http://www.swift.ac.uk/user_objects/

http://www.swift.ac.uk/analysis/xrt/digest_cal.php
} 


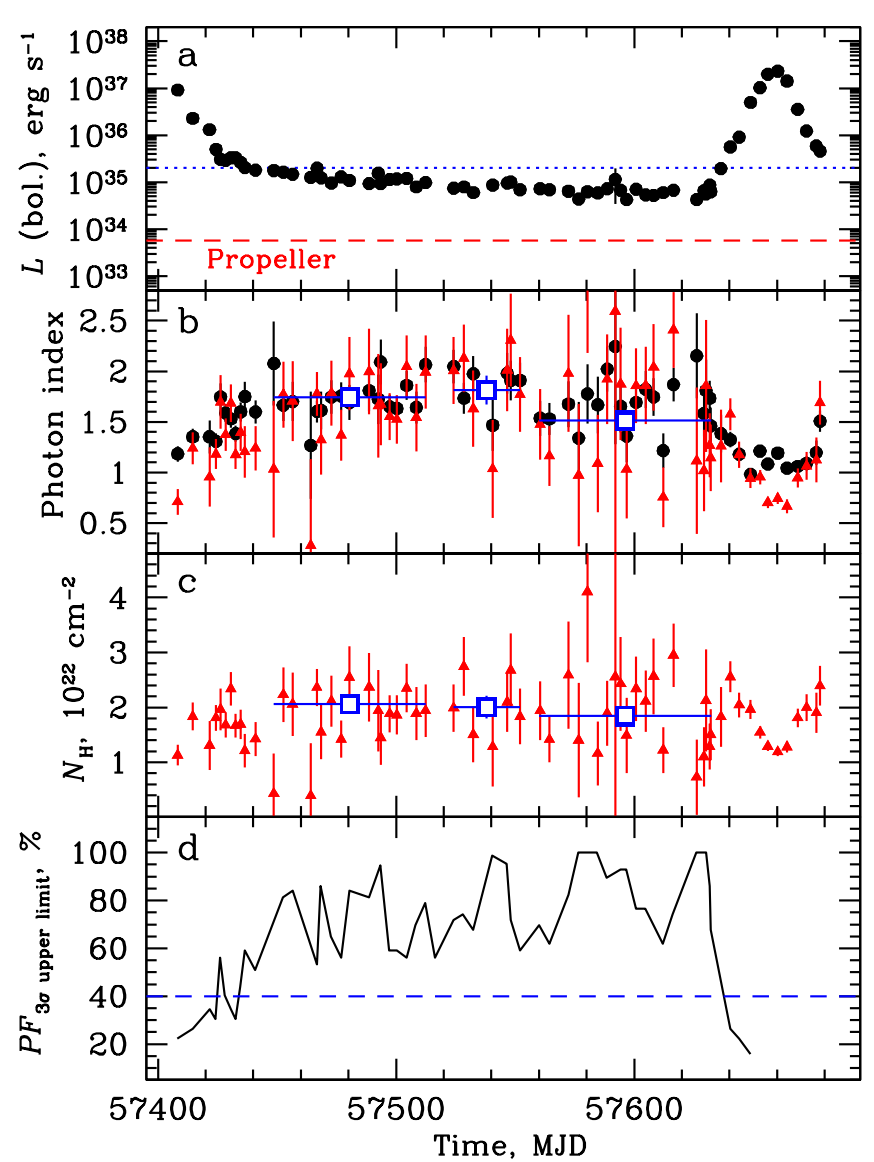

Fig. 1. Panel a: Bolometric luminosity of GRO J1008-57 obtained by the Swift/XRT telescope. The luminosity is calculated from the unabsorbed flux assuming a distance $d=5.8 \mathrm{kpc}$ and fixed $N_{\mathrm{H}}=$ $2.0 \times 10^{22} \mathrm{~cm}^{-2}$. The dashed red and dotted blue lines show the predicted luminosity $L_{\text {prop }}$ for the transition to the propeller regime and the observed transition to the low-level stable accretion regime, respectively. Panels $b$ and $c$ represent variations of the photon index and absorption value with time, respectively. Black symbols correspond to the fixed $N_{\mathrm{H}}$, red triangles and blue open squares to the absorption as a free parameter. In panel $d$ the estimated $3 \sigma$ upper limits for the pulsed fraction as function of time are shown for the Swift/XRT data with the horizontal dashed line showing the typical pulsed fraction measured during the bright state. The pulsations are clearly detected in all observations, which are expected to be sufficiently sensitive (i.e. below this line), but not elsewhere.

described in earlier reports (Kühnel et al. 2013; Bellm et al. 2014), the pulse profile exhibits two peaks with a relatively high pulsed fraction of $40-50 \%$, as is typical for most of XRPs (Lutovinov \& Tsygankov 2009). However, no pulsations were detected after the observation 00031030034 (MJD 57430.51), when the source entered the low-level stable accretion state. On the other hand, the number of photons per observation becomes too low at this stage (a few tens to a few hundreds), so that the non-detection of the pulsations might simply be due to the insufficient counting statistics. For all low-level observations, the $3 \sigma$ upper limits for the amplitude of the pulsations calculated based on the H-test (de Jager et al. 1989) and following the approach described in Brazier (1994) indeed lie in range 40-95\%, as illustrated in Fig. 1d. The non-detection of the pulsations in individual Swift observations at low fluxes is therefore expected, taking their sensitivity to pulsed flux into account.

On the other hand, a search for the pulsations in the combined light-curve is complicated by the sparse sampling of the light curve and the uncertainty in the orbital parameters of the system. We therefore conclude that the non-detection of the pulsations by Swift does not contradict a presence of pulsations given the available counting statistics and light-curve sampling.

Somewhat stronger limits on the amplitude of the pulsations in the quiescence can be obtained from the $5 \mathrm{ks}$ long Chandra observation 14639 of the source carried out in May 2013 (MJD 56440.72) between the Type I outbursts. The bolometric luminosity calculated in the same way as for Swift data is $\sim 10^{35} \mathrm{erg} \mathrm{s}^{-1}$, which is comparable with the values measured by Swift during our observations in the low-level stable state. To search for the pulsations, we first extracted photons from a circle with radius $7.4^{\prime \prime}$ centred on the source position after applying the standard screening criteria described in the instrument documentation. This yields 3215 source photons, which is comparable to the yield for individual Swift/XRT observations during the outburst and only a factor of two less than the total number of photons detected by Swift in the low-level state. On the other hand, the Chandra observation is not affected by the uncertainty on the orbital parameters. To search for the pulsations, we first corrected the photon arrival times for the effects of orbital motion in solar and binary systems using the ephemeris reported by Kühnel et al. (2013) and then used the same approach as for the Swift data. No significant pulsations around the source pulse frequency could be detected with the $3 \sigma$ upper limit of $\sim 20 \%$ for periods in the range $80-100 \mathrm{~s}$. We note, however, that Chandra data are susceptible to pile-up, which potentially reduces the sensitivity to pulsed flux. The pile-up fraction is lower for pixels in the wings of the point-spread function and higher in its core, meaning that it depends on the count-rate in a given pixel. Therefore we repeated the simulation above for each pixel within a circle with radius of 10 pixels centred on the source. All events detected within a single frame (3.2 s) were considered as one event and noted to estimate the total pile-up fraction, which is around $15 \%$. The upper limit on the pulsed fraction was then estimated using a concatenated event list from all pixels as described above, and it indeed was significantly higher at $\sim 35 \%$. While slightly lower than the typical pulsed fraction observed during the outburst, this by no means excludes pulsations at low fluxes, especially considering the fact that the pulsed fraction is known to be variable and that we ignored the background for this estimate. We therefore conclude that additional observations, preferably with $X M M-N e w t o n$, are required in order to study the pulsations in the low-level stable accretion regime.

\subsection{Spectral variability}

All spectra from individual observations can be fitted with a simple absorbed power-law model. To illustrate the stability of the spectral shape over the luminosity and time, in Fig. 2 we present three spectra of GRO J1008-57 obtained in the brightest state of the January 2016 outburst (ObsId 00031030028; red circles), around the transition luminosity (ObsId 00031030035 ; blue squares), and deep inside the stable low-level state (ObsIds $00031030066-85$; magenta crosses) with luminosities $6.9 \times 10^{36}$, $3.1 \times 10^{35}$, and $5.7 \times 10^{34} \mathrm{erg} \mathrm{s}^{-1}$, respectively. No significant differences are immediately suggested by the data.

A more detailed analysis, however, reveals a minor change in the absorption and photon index values in the data collected in the bright (outburst) and low-level states. To obtain sufficient count statistics in the low-level state (between MJD 57440 and 57630 ), we binned the available data into three wide bins (see Fig. 1 and Table A.1). 


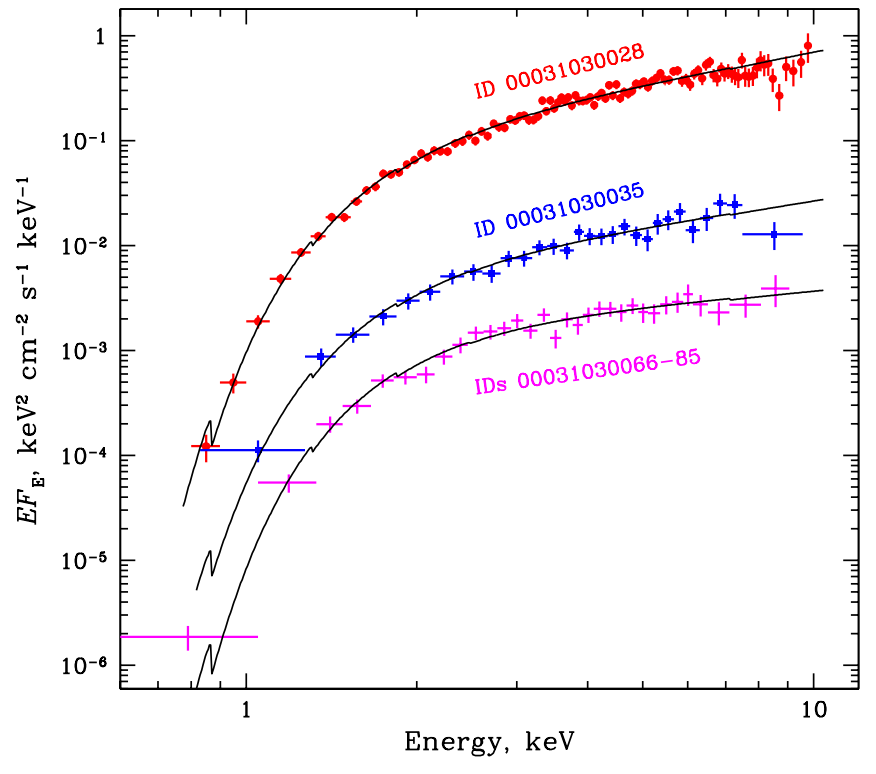

Fig. 2. Spectra of GRO J1008-57 obtained by Swift/XRT in different luminosity states: the brightest state of the January 2016 outburst (ObsId 00031030028; red circles), the transiting luminosity (ObsId 00031030035; blue squares), and deep inside the stable low-level state (ObsIds 00031030066-85; magenta crosses).

As a result, we found that during the outburst (before MJD 57440 and after MJD 57648) the mean value of $N_{\mathrm{H}}$ is lower than $\sim 1.8 \times 10^{22} \mathrm{~cm}^{-2}$, which is just slightly higher than the Galactic value in this direction $\left(N_{\mathrm{H}}=1.4 \times 10^{22} \mathrm{~cm}^{-2}\right.$; Kalberla et al. 2005). On the other hand, after the transition its value increases up to $N_{\mathrm{H}}=(2.0 \pm 0.1) \times 10^{22} \mathrm{~cm}^{-2}$. The difference is most prominent in the parameters of the averaged spectra. The variability of the spectral parameters during the whole orbital cycle of GRO J1008-57 is shown in Fig. 1 for single and averaged observations. The middle and bottom panels illustrate variations in photon index and absorption column with time, respectively. Black symbols correspond to the absorption column fixed at $N_{\mathrm{H}}=2.0 \times 10^{22} \mathrm{~cm}^{-2}$, while red triangles and blue open squares show the fits with free absorption.

The observational log as well as the best-fit spectral parameters for the single and averaged observations are presented in Table A.1 for the free and fixed absorption values.

\section{Discussion}

Accreting magnetized neutron stars (XRPs, accreting millisecond pulsars, and accreting magnetars) exhibit a complex behaviour that is determined by the interaction of their magnetospheres with the accreting matter. Particularly, observational evidence for the propeller effect was found in a number of sources with magnetic fields from $\sim 10^{8} \mathrm{G}$ (for the accreting millisecond pulsar SAX J1808.4-3658; Campana et al. 2008) to $\sim 10^{14} \mathrm{G}$ (for the first pulsating ULX M82 X-2; Tsygankov et al. 2016b). The XRPs typically have magnetic fields somewhere in between these values, and some of them also exhibit a transition to the propeller regime (Stella et al. 1986; Cui 1997; Campana et al. 2001; Tsygankov et al. 2016a; Lutovinov et al. 2017).

Our observational campaign was originally aimed at detecting the transition of GRO J1008-57 to the propeller regime. In the case of GRO J1008-57, Eq. (4) gives $L_{\text {prop }}=$ $5.6 \times 10^{33} \mathrm{erg} \mathrm{s}^{-1}$. However, instead of a sharp drop in flux, GRO J1008-57 entered a stable accretion regime at a luminosity of about $10^{35} \mathrm{erg} \mathrm{s}^{-1}$ (see Fig. 1), about one and a half orders of magnitude higher than $L_{\text {prop }}$.

We note that unlike $\mathrm{V} \quad 0332+53$ and $4 \mathrm{U} \quad 0115+63$, where an obvious spectral softening was observed after the transition to the propeller regime (Tsygankov et al. 2016a; Wijnands \& Degenaar 2016), no such changes have been observed in GRO J1008-57 after the transition to the stable accretion state. This strongly suggests that the emission mechanism remains the same, that is to say, the source continues to accrete even though the data quality was insufficient for detecting pulsations.

\subsection{Accretion disc stability}

As was shown by Lasota (1997), stable accretion onto the compact object is possible when the mass accretion rate is high enough to keep the entire accretion disc hot and fully ionised (temperature $>6500 \mathrm{~K}$ ) or when the mass accretion rate is so low that the temperature is below the critical value even at the inner radius. At intermediate levels of the mass accretion rate, the appearance of the region with a temperature of $6500 \mathrm{~K}$ will cause a rapidly propagating heating or cooling front through the disc, leading to the fast variability of the source flux. In this section we place the XRPs into the context of the disc instability model and make predictions for pulsars with different properties (see also the discussion in Tsygankov et al. 2016a).

We consider conditions under which an XRP may accrete matter from the recombined (cold) disc. Particularly, two criteria have to be fulfilled: (1) the mass accretion rate has to be high enough to allow the matter to penetrate the centrifugal barrier, and at the same time, (2) the mass accretion rate has to be sufficiently low to allow the disc to remain sufficiently cold $(<6500 \mathrm{~K})$ at every radius.

The first criterion was discussed above and is realized when the magnetospheric radius $R_{\mathrm{m}}$ is smaller than the corotation radius $R_{\mathrm{c}}$ :

$R_{\mathrm{m}} \leq R_{\mathrm{c}}$.

Condition (5) is fulfilled when the accretion luminosity is higher than the threshold luminosity presented above (see Eq. (4)): $L \geq$ $L_{\text {prop }}$.

The second criterion is satisfied when the mass accretion rate is below the value given by Eq. (1) ( $\dot{M}<\dot{M}_{\text {cold }}$; Lasota 1997). Substituting the magnetospheric radius instead of $r$ into Eq. (1), we obtain another condition for the stable accretion from a cold disc:

$L<L_{\text {cold }}=9 \times 10^{33} k^{1.5} M_{1.4}^{0.28} R_{6}^{1.57} B_{12}^{0.86} \mathrm{erg} \mathrm{s}^{-1}$.

Below this level, the temperature in the accretion disc is lower than $6500 \mathrm{~K}$ at $R>R_{\mathrm{m}}$.

If the threshold luminosity for the propeller regime is higher than the luminosity that corresponds to the transition to the cold $\operatorname{disc}\left(L_{\text {prop }}>L_{\text {cold }}\right)$, then the decrease in mass accretion rate during the outburst decay will cause the transition to the propeller state. In the opposite case $\left(L_{\text {prop }}<L_{\text {cold }}\right)$, the accretion disc will switch to the cold state with low viscosity, allowing stable accretion even at a very low rate.

Interestingly, the final state of the source after an outburst is determined by two fundamental parameters of the neutron star: the magnetic field, and the spin period. Equating the expressions for luminosities $L_{\text {cold }}$ and $L_{\text {prop }}$, we can derive the critical value of the spin period as a function of the neutron star magnetic field:

$P^{*}=36.6 k^{6 / 7} B_{12}^{0.49} M_{1.4}^{-0.17} R_{6}^{1.22} \mathrm{~s}$. 
If the spin period $P<P^{*}$, a pulsar will end up in the propeller regime. Otherwise, the source will start to accrete stably from the cold disc.

\subsection{More accurate condition for accretion from a cold disc}

The condition given by Eq. (6) is based on the assumption that the disc temperature is highest at the inner radius given by $R_{\mathrm{m}}$. However, an effective temperature distribution over the radial coordinate depends on the exact boundary condition and viscous stress at the magnetospheric radius. Condition (6) can be derived more accurately. There is an uncertainty in the location, where the stress disappears. This uncertainty is described by the parameter $\beta: \beta=1$ corresponds to a disappearance of the stress at the magnetospheric radius $R_{\mathrm{m}}$, while $\beta=0$ corresponds to the case when the stress disappears at $r \ll R_{\mathrm{m}}$. Then the distribution of effective temperature $T_{\text {eff }}$ over the radial coordinate is given by (Frank et al. 2002)

$\sigma_{\mathrm{SB}} T_{\mathrm{eff}}^{4}=\frac{3}{8 \pi} \frac{L}{r^{3}} R\left[1-\beta\left(\frac{R_{\mathrm{m}}}{r}\right)^{1 / 2}\right]$,

where $\sigma_{\mathrm{SB}}$ is the Stefan-Boltzmann constant. The maximum effective temperature,

$\sigma_{\mathrm{SB}} T_{\mathrm{eff}, \max }^{4}=A \frac{3}{8 \pi} \frac{L}{R_{\mathrm{m}}^{3}} R$,

is achieved at the radius

$r_{\max }= \begin{cases}\frac{49}{36} \beta^{2} R_{\mathrm{m}}, & \text { if } \beta \geq \frac{\sqrt{3}}{2}, \\ R_{\mathrm{m}}, & \text { if } \beta<\frac{\sqrt{3}}{2},\end{cases}$

where

$A= \begin{cases}0.057 \beta^{-6}, & \text { if } \beta \geq \frac{\sqrt{3}}{2}, \\ 1-\beta, & \text { if } \beta<\frac{\sqrt{3}}{2} .\end{cases}$

As a result, condition (6) can be rewritten in more accurate way. In this approach the maximum effective temperature in the disc is lower than $6500 \mathrm{~K}$ throughout the disc when the accretion luminosity is

$L \leq L_{\text {cold }}^{(2)} \simeq 7 \times 10^{33} A^{-7 / 13} k^{21 / 13} M_{1.4}^{3 / 13} R_{6}^{23 / 13} B_{12}^{12 / 13} T_{6500}^{28 / 13} \mathrm{erg} \mathrm{s}^{-1}$,

where $T_{6500}=T_{\text {eff }} / 6500 \mathrm{~K}$. This estimate is more accurate than Eq. (6) as it accounts for interaction of the disc with the magnetosphere. In the case when the stress disappears at $R_{\mathrm{m}}$, condition (12) gives $L_{\text {cold }}$ higher by a factor of a few than Eq. (6).

As can be seen from Eq. (12), our prediction of the transition to the accretion from the cold disc $\left(L_{\text {cold }}^{(2)} \sim 7 \times 10^{34} \mathrm{erg} \mathrm{s}^{-1}\right)$ coincides with the observed value within the factor of $2-3$. Taking into account existing systematic uncertainties (the distance to the system, the physics of the coupling between the disc and the star, the width of the coupling region, and the uncertainty in the transiting temperature), we consider this match as support of our physical picture.

Another source of uncertainty can be associated with the irradiation of the accretion disc by the central object. Some theoretical models have indeed explained the long duration of the soft $\mathrm{X}$-ray transients by irradiation of the outer regions of the accretion disc, which keeps them in hot ionised state for a longer time (see, e.g. King \& Ritter 1998). There are also models of the soft
X-ray transient bursts that do not need external irradiation for explanation of accretion discs dynamics (see, e.g. Lipunova 2015, and references therein), which might be more appropriate for our case. The observed outburst duration seems to be compatible with the cooling-wave propagation timescale. Indeed, the accretion disc is expected to extend to $4 \times 10^{10} \mathrm{~cm}$ close to the peak of the outburst (assuming an effective temperature $T_{\text {eff }}=6500 \mathrm{~K}$ at this radius). The cooling-wave velocity $V_{\mathrm{cw}}$ is a few times lower than the heating wave velocity $V_{\mathrm{hw}}$ (Cannizzo et al. 1988), which can be estimated as $V_{\mathrm{hw}} \approx \alpha V_{\mathrm{s}}$, where $V_{\mathrm{s}} \approx 10^{6} \mathrm{~cm} \mathrm{~s}^{-1}$ is the sound speed (Meyer 1984; Cannizzo 1993). The propagation time of the cooling wave from the radius $4 \times 10^{10} \mathrm{~cm}$ inward to $R_{\mathrm{m}}$ is therefore expected to be about 10-20 days (assuming $\alpha=0.1$ ), which is comparable with observed duration.

Using the updated Eq. (12) for the critical accretion luminosity $L_{\text {cold }}^{(2)}$, we can derive an updated equation for the critical value of the spin period $P^{*}$, which, as described above, determines the pulsar behaviour at very low mass-accretion rate. Now instead of Eq. (7) we have

$P^{*}=40.7 k^{21 / 23} B_{12}^{6 / 13} M_{1.4}^{-5 / 13} R_{6}^{18 / 13} A^{3 / 13} T_{6500}^{-12 / 13} \mathrm{~s}$.

This equation can be used to predict the behaviour of any XRP (with a known magnetic field) expected in the case of transient activity. This is especially important for XRPs in Be binary systems (BeXRPs), which are known to be transient sources (see, e.g. Reig 2011) with magnetic fields measured from the cyclotron lines in their spectra (Walter et al. 2015). For illustration, we show some known BeXRPs as well as the accreting millisecond pulsar SAX J1808.4-3658, the intermediate pulsar GRO J1744-28, and the accreting magnetar M82 X-2 on the $B-P$ plane in Fig. 3. All sources in this plane are divided with the prediction from Eq. (13) into two groups: (i) those entering the propeller regime at a low mass-accretion rate (below the line), and (ii) sources where stable accretion from the cold disc continues at any accretion rate (above the line). Persistent low-luminous BeXRPs (Reig \& Roche 1999) are shown in green. Clearly, GRO J1008-57 resides in the area corresponding to the sources with accretion from the cold disc. The majority of sources residing below the $P^{*}(B)$ line have previously been shown to exhibit transitions to the propeller regime (for a review see Tsygankov et al. 2016a). It is important to note that peak luminosities in BeXRPs outbursts are ranging from $10^{37}$ to $10^{39} \mathrm{erg} \mathrm{s}^{-1}$, exceeding all possible values of $L_{\text {cold }}$ and $L_{\text {prop }}$. During the outburst decline, such sources therefore inevitably end up in one of the above-mentioned states.

\section{Conclusion}

We analysed the Swift/XRT observations of GRO J1008-57 obtained between two subsequent Type I outbursts in January and September 2016. The original idea was to detect the transition of the source to the propeller regime, which is accompanied by an abrupt decrease in source flux and a softening of its spectrum. However, during the declining phase of the outburst, the source unexpectedly stopped its fading and entered a stable accretion state that was characterised by an accretion rate of the order of $\sim 10^{14}-10^{15} \mathrm{~g} \mathrm{~s}^{-1}$ and a hard spectrum. We associate this state with accretion from the cold (low-ionised) disc with a temperature below $\sim 6500 \mathrm{~K}$.

We proposed a model of accretion from the cold disc in the systems harbouring neutron stars with strong magnetic fields (i.e. XRPs). The basic idea of the model is that in slowly rotating neutron stars the centrifugal barrier caused by the rotating 


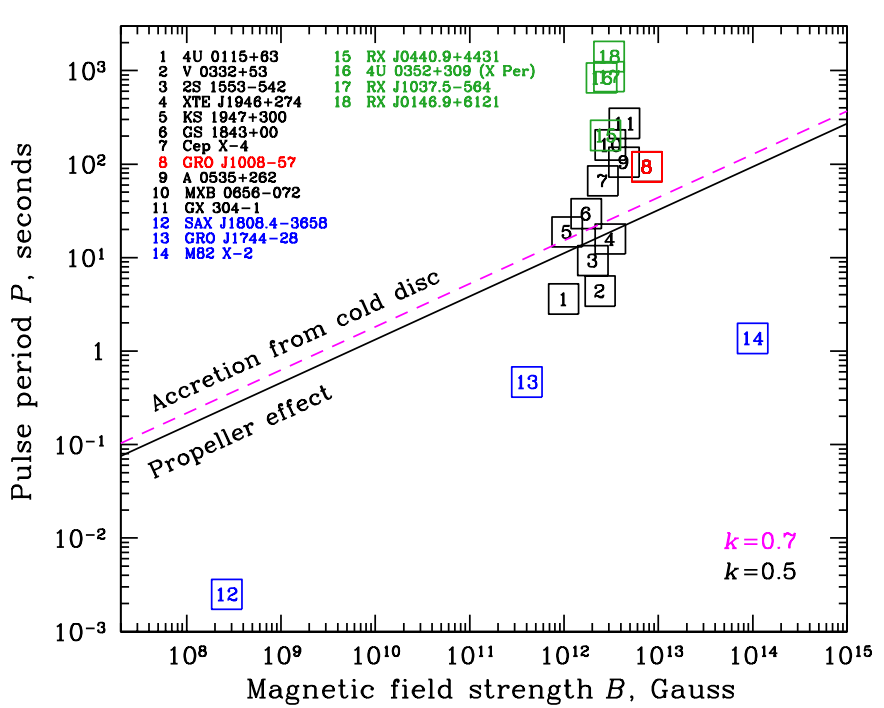

Fig. 3. Collection of some known BeXRPs (shown in black), as well as the accreting millisecond pulsar SAX J1808.4-3658, the intermediate pulsar GRO J1744-28, and the accreting magnetar M82 X-2 (all three shown in blue) on the $B-P$ plane. The solid and dashed lines correspond to the prediction of $P^{*}(B)$ from Eq. (13) for $\beta=1$ and two different values of parameter $k: 0.5$ and 0.7 , respectively. This line separates sources where the propeller regime is possible from sources with stable accretion from the cold disc. Persistent low-luminous BeXRPs are shown in green. GRO J1008-57 is shown in red and resides in the area corresponding to the sources with accretion from the cold disc.

magnetosphere is greatly suppressed in comparison to the fast rotating neutron stars, leading to a much lower threshold luminosity for the transition to the propeller regime. This allows such sources to reach mass-accretion rates that are so low that the temperature throughout the accretion disc becomes lower than the hydrogen recombination limit of $\sim 6500 \mathrm{~K}$.

When this mass accretion rate is reached, the fast fading of the source intensity should stop and further accretion with a low rate in the stable accretion regime from the cold disc with very low viscosity is expected to continue. This behaviour was observed in the pulsar GRO J1008-57 between two consequent Type I outbursts in January and September 2016.

Our model has strong predictive power. Particularly, the transition to the accretion regime from a cold disc is expected to be observed in all XRPs with a certain combination of pulse period and magnetic field strength. Other manifistations of the cold disc accretion associated with a change in disc structure could be anticipated. A change of the inner disc radius can be expected to affect the spin evolution of the pulsar, aperiodic variability properties, pulse profiles, and the energy spectrum of the source. A detailed calculation of the accretion disc structure in this case is ongoing and will be published elsewhere.

Acknowledgements. We are grateful to Ilia Potravnov for a number of usefu comments and to the Swift team for the execution of our ToO request. This work was supported by the Russian Science Foundation grant 14-12-01287 (S.S.T., A.A.M., A.A.L., V.S.), the Academy of Finland grant 268740, and the National Science Foundation grant PHY-1125915 (J.P.). V.D. thanks the Deutsches Zentrums for Luft- und Raumfahrt (DLR) and the DFG for financial support (grant DLR 50 OR 0702). We also acknowledge the support of COST Action MP1304.

\section{References}

Bellm, E. C., Fürst, F., Pottschmidt, K., et al. 2014, ApJ, 792, 108 Brazier, K. T. S. 1994, MNRAS, 268, 709

Burrows, D. N., Hill, J. E., Nousek, J. A., et al. 2005, Space Sci. Rev., 120, 165 Campana, S., Gastaldello, F., Stella, L., et al. 2001, ApJ, 561, 924

Campana, S., Stella, L., \& Kennea, J. A. 2008, ApJ, 684, L99 Cannizzo, J. K. 1993, ApJ, 419, 318

Cannizzo, J. K., Shafter, A. W., \& Wheeler, J. C. 1988, ApJ, 333, 227

Cash, W. 1979, ApJ, 228, 939

Chen, W., Shrader, C. R., \& Livio, M. 1997, ApJ, 491, 312

Cheng, F. H., Horne, K., Panagia, N., et al. 1992, ApJ, 397, 664

Cherepashchuk, A. M. 2000, Space Sci. Rev., 93, 473

Coe, M. J., Bird, A. J., Hill, A. B., et al. 2007, MNRAS, 378, 1427

Cui, W. 1997, ApJ, 482, L163

de Jager, O. C., Raubenheimer, B. C., \& Swanepoel, J. W. H. 1989, A\&A, 221, 180

Evans, P. A., Beardmore, A. P., Page, K. L., et al. 2009, MNRAS, 397, 1177

Frank, J., King, A., \& Raine, D. J. 2002, Accretion Power in Astrophysics, third edition (Cambridge, UK: Cambridge University Press), 398

Gehrels, N., Chincarini, G., Giommi, P., et al. 2004, ApJ, 611, 1005

Ghosh, P., \& Lamb, F. K. 1978, ApJ, 223, L83

Illarionov, A. F., \& Sunyaev, R. A. 1975, A\&A, 39, 185

Kalberla, P. M. W., Burton, W. B., Hartmann, D., et al. 2005, A\&A, 440, 775

King, A. R., \& Ritter, H. 1998, MNRAS, 293, L42

Kretschmar, P., Kuehnel, M., Nakajima, M., et al. 2016, ATel, 8547

Kuehnel, M., Mueller, S., Kreykenbohm, I., et al. 2012, ATel, 4564

Kühnel, M., Müller, S., Kreykenbohm, I., et al. 2013, A\&A, 555, A95

Lasota, J. P. 1997, in Accretion Phenomena and Related Outflows, eds. D. T.

Wickramasinghe, G. V. Bicknell, \& L. Ferrario, IAU Colloq., 163, ASP Conf. Ser., 121, 351

Lasota, J.-P. 2001, New Astron. Rev., 45, 449

Levine, A. M., \& Corbet, R. 2006, ATel, 940

Lipunova, G. V. 2015, ApJ, 804, 87

Lutovinov, A. A., \& Tsygankov, S. S. 2009, Astron. Lett., 35, 433

Lutovinov, A. A., Tsygankov, S. S., Krivonos, R. A., Molkov, S. V., \& Poutanen, J. 2017, ApJ, 834, 209

Meyer, F. 1984, A\&A, 131, 303

Meyer, F., \& Meyer-Hofmeister, E. 1984, A\&A, 132, 143

Mineshige, S., Yamasaki, T., \& Ishizaka, C. 1993, PASJ, 45, 707

Nakajima, M., Kawase, T., Negoro, H., et al. 2016, ATel, 9512

Reig, P. 2011, Ap\&SS, 332, 1

Reig, P., \& Roche, P. 1999, MNRAS, 306, 100

Riquelme, M. S., Torrejón, J. M., \& Negueruela, I. 2012, A\&A, 539, A114

Shrader, C. R., Sutaria, F. K., Singh, K. P., \& Macomb, D. J. 1999, ApJ, 512, 920

Smak, J. 1984, Acta Astron., 34, 161

Stella, L., White, N. E., \& Rosner, R. 1986, ApJ, 308, 669

Stollberg, M. T., Finger, M. H., Wilson, R. B., et al. 1993, IAU Circ., 5836

Tsygankov, S. S., Lutovinov, A. A., Doroshenko, V., et al. 2016a, A\&A, 593, A16

Tsygankov, S. S., Mushtukov, A. A., Suleimanov, V. F., \& Poutanen, J. 2016b, MNRAS, 457, 1101

Walter, R., Lutovinov, A. A., Bozzo, E., \& Tsygankov, S. S. 2015, A\&ARv, 23,

Warner, B. 2003, Cataclysmic Variable Stars (Cambridge: Cambridge University Press), 592

Wijnands, R., \& Degenaar, N. 2016, MNRAS, 463, L46

Yamamoto, T., Mihara, T., Sugizaki, M., et al. 2013, ATel, 4759, 1 


\section{Appendix A: Additional table}

Table A.1. Swift/XRT observations of GRO J1008-57.

\begin{tabular}{|c|c|c|c|c|c|c|}
\hline \multirow[t]{2}{*}{ Obs Id } & \multirow{2}{*}{$\begin{array}{l}\text { Date } \\
\text { MJD }\end{array}$} & \multirow{2}{*}{$\begin{array}{c}\text { Exposure } \\
\text { ks }\end{array}$} & \multicolumn{2}{|c|}{$N_{\mathrm{H}}=2.0 \times 10^{22} \mathrm{~cm}^{-2}$} & \multicolumn{2}{|c|}{ Free $N_{\mathrm{H}}$} \\
\hline & & & $\begin{array}{c}\text { XRT flux } \\
{ }^{a} \\
10^{-11} \mathrm{erg} \mathrm{s}^{-1} \mathrm{~cm}^{-2}\end{array}$ & $\Gamma^{b}$ & $\begin{array}{c}N_{\mathrm{H}} \\
10^{22} \mathrm{~cm}^{-2}\end{array}$ & $\Gamma^{b}$ \\
\hline 00031030028 & 57408.1683 & 1.0 & $142 \pm 5$ & $1.2 \pm 0.1$ & $1.1 \pm 0.2$ & $0.7 \pm 0.1$ \\
\hline 00031030029 & 57414.4910 & 1.3 & $35.5 \pm 1.3$ & $1.4 \pm 0.1$ & $1.8 \pm 0.3$ & $1.2 \pm 0.2$ \\
\hline 00031030030 & 57421.6127 & 1.2 & $20.3 \pm 1.5$ & $1.4 \pm 0.2$ & $1.3 \pm 0.4$ & $1.0 \pm 0.3$ \\
\hline 00031030031 & 57424.0809 & 2.5 & $7.8 \pm 0.4$ & $1.3 \pm 0.1$ & $1.8 \pm 0.2$ & $1.2 \pm 0.1$ \\
\hline 00031030032 & 57426.0158 & 1.2 & $4.8_{-0.2}^{+0.3}$ & $1.7 \pm 0.1$ & $2.0 \pm 0.4$ & $1.7 \pm 0.3$ \\
\hline 00031030033 & 57428.0596 & 2.3 & $4.6_{-0.1}^{+0.2}$ & $1.6 \pm 0.1$ & $1.7 \pm 0.2$ & $1.4 \pm 0.2$ \\
\hline 00031030034 & 57430.5115 & 2.7 & $5.1_{-0.1}^{+0.1}$ & $1.5 \pm 0.1$ & $2.3 \pm 0.3$ & $1.7 \pm 0.2$ \\
\hline 00031030035 & 57432.5746 & 2.9 & $5.1_{-0.1}^{-0.1}$ & $1.4 \pm 0.1$ & $1.7 \pm 0.2$ & $1.2 \pm 0.1$ \\
\hline 00031030036 & 57434.5145 & 2.5 & $4.1 \pm 0.2$ & $1.6 \pm 0.1$ & $1.7 \pm 0.3$ & $1.4 \pm 0.2$ \\
\hline 00031030037 & 57436.4258 & 1.3 & $3.2 \pm 0.2$ & $1.7 \pm 0.1$ & $1.2 \pm 0.3$ & $1.2 \pm 0.3$ \\
\hline 00031030038 & 57440.8239 & 2.3 & $2.8 \pm 0.1$ & $1.6 \pm 0.1$ & $1.4 \pm 0.3$ & $1.2 \pm 0.2$ \\
\hline 00031030040 & 57448.6581 & 0.2 & $2.8_{-0.5}^{+0.8}$ & $2.1 \pm 0.4$ & $0.4 \pm 0.7$ & $1.0 \pm 0.7$ \\
\hline 00031030041 & 57452.4376 & 1.3 & $2.5 \pm 0.2$ & $1.7 \pm 0.2$ & $2.2 \pm 0.5$ & $1.8 \pm 0.3$ \\
\hline 00031030042 & 57456.4917 & 1.2 & $2.3 \pm 0.2$ & $1.7 \pm 0.2$ & $2.1 \pm 0.6$ & $1.7 \pm 0.4$ \\
\hline 00031030043 & 57464.1391 & 0.2 & $2.0_{-0.4}^{+0.5}$ & $1.3 \pm 0.5$ & $0.4 \pm 1.0$ & $0.3 \pm 0.9$ \\
\hline 00031030044 & 57466.6732 & 2.4 & $3.1_{-0.1}^{+0.4}$ & $1.6 \pm 0.1$ & $2.4 \pm 0.3$ & $1.8 \pm 0.2$ \\
\hline 00031030045 & 57468.2726 & 1.3 & $1.9_{-0.2}^{+0.1}$ & $1.6 \pm 0.2$ & $1.6 \pm 0.5$ & $1.3 \pm 0.3$ \\
\hline 00031030046 & 57472.5946 & 2.4 & $1.5 \pm 0.1$ & $1.7 \pm 0.2$ & $2.1 \pm 0.5$ & $1.8 \pm 0.3$ \\
\hline 00031030047 & 57476.8448 & 2.6 & $2.0 \pm 0.1$ & $1.8 \pm 0.1$ & $1.4 \pm 0.3$ & $1.4 \pm 0.3$ \\
\hline 00031030048 & 57480.1727 & 1.8 & $1.7_{-0.1}^{+0.2}$ & $1.7 \pm 0.2$ & $2.5 \pm 0.6$ & $2.0 \pm 0.4$ \\
\hline 00031030049 & 57488.6718 & 1.3 & $1.5 \pm 0.1$ & $1.8 \pm 0.2$ & $2.4 \pm 0.6$ & $2.0 \pm 0.4$ \\
\hline 00031030050 & 57492.4004 & 0.6 & $2.4_{-0.3}^{+0.2}$ & $1.7 \pm 0.2$ & $1.9 \pm 0.7$ & $1.7 \pm 0.5$ \\
\hline 00031030051 & 57493.4566 & 1.3 & $1.5 \pm 0.2$ & $2.1 \pm 0.2$ & $1.4 \pm 0.5$ & $1.7 \pm 0.4$ \\
\hline 00031030052 & 57497.0396 & 3.1 & $1.8 \pm 0.1$ & $1.6 \pm 0.1$ & $1.9 \pm 0.3$ & $1.6 \pm 0.2$ \\
\hline 00031030053 & 57500.2303 & 2.9 & $1.8 \pm 0.1$ & $1.6 \pm 0.1$ & $1.9 \pm 0.4$ & $1.5 \pm 0.2$ \\
\hline 00031030054 & 57504.3630 & 2.2 & $1.9 \pm 0.1$ & $1.9 \pm 0.1$ & $2.4 \pm 0.4$ & $2.0 \pm 0.3$ \\
\hline 00031030055 & 57508.1415 & 2.9 & $1.2 \pm 0.1$ & $1.6 \pm 0.2$ & $1.9 \pm 0.5$ & $1.5 \pm 0.3$ \\
\hline 00031030056 & 57512.2662 & 1.9 & $1.5_{-0.1}^{+0.2}$ & $2.1 \pm 0.2$ & $1.9 \pm 0.5$ & $2.0 \pm 0.4$ \\
\hline 00031030058 & 57524.0342 & 2.8 & $1.2 \pm 0.1$ & $2.0 \pm 0.2$ & $2.0 \pm 0.4$ & $2.0 \pm 0.3$ \\
\hline 00031030059 & 57528.0203 & 2.9 & $1.2 \pm 0.1$ & $1.7 \pm 0.2$ & $2.7 \pm 0.5$ & $2.1 \pm 0.3$ \\
\hline 00031030060 & 57532.4048 & 2.9 & $0.9 \pm 0.1$ & $2.0 \pm 0.2$ & $1.5 \pm 0.5$ & $1.6 \pm 0.4$ \\
\hline 00031030061 & 57540.3100 & 1.1 & $1.4_{-0.2}^{+0.1}$ & $1.5 \pm 0.3$ & $1.3 \pm 0.7$ & $1.0 \pm 0.5$ \\
\hline 00031030062 & 57546.4969 & 1.5 & $1.5 \pm 0.1$ & $2.0 \pm 0.2$ & $2.1 \pm 0.5$ & $2.0 \pm 0.4$ \\
\hline 00031030063 & 57548.0885 & 1.6 & $1.6_{-01}^{+0.2}$ & $1.9 \pm 0.2$ & $2.7 \pm 0.7$ & $2.3 \pm 0.5$ \\
\hline 00031030064 & 57552.0065 & 2.9 & $1.1 \pm 0.1$ & $1.9 \pm 0.2$ & $1.8 \pm 0.5$ & $1.8 \pm 0.4$ \\
\hline 00031030066 & 57560.0381 & 2.6 & $1.1 \pm 0.1$ & $1.5 \pm 0.2$ & $1.9 \pm 0.5$ & $1.5 \pm 0.3$ \\
\hline 00031030067 & 57564.2988 & 3.0 & $1.1 \pm 0.1$ & $1.5 \pm 0.2$ & $1.4 \pm 0.4$ & $1.2 \pm 0.3$ \\
\hline 00031030068 & 57572.2871 & 1.7 & $1.0 \pm 0.1$ & $1.7 \pm 0.2$ & $2.6 \pm 1.0$ & $2.0 \pm 0.6$ \\
\hline 00031030069 & 57576.4712 & 1.6 & $0.7 \pm 0.1$ & $1.3 \pm 0.4$ & $1.4 \pm 1.0$ & $1.0 \pm 0.7$ \\
\hline 00031030070 & 57580.3262 & 1.0 & $1.0 \pm 0.1$ & $1.8 \pm 0.3$ & $4.1 \pm 1.3$ & $2.9 \pm 0.7$ \\
\hline 00031030071 & 57584.3187 & 1.3 & $0.9 \pm 0.1$ & $1.7 \pm 0.3$ & $1.2 \pm 0.6$ & $1.1 \pm 0.5$ \\
\hline 00031030072 & 57588.4948 & 1.6 & $1.1 \pm 0.1$ & $2.0 \pm 0.2$ & $1.9 \pm 0.6$ & $1.9 \pm 0.4$ \\
\hline 00031030073 & 57592.0136 & 0.1 & $1.8_{-0.7}^{+1.8}$ & $2.2 \pm 1.0$ & $2.6 \pm 3.2$ & $2.6 \pm 2.4$ \\
\hline 00031030074 & 57594.2706 & 1.4 & $1.1 \pm 0.1$ & $1.7 \pm 0.3$ & $2.4 \pm 0.9$ & $1.9 \pm 0.6$ \\
\hline 00031030075 & 57596.7272 & 2.7 & $0.66_{-0.07}^{+0.08}$ & $1.4 \pm 0.3$ & $1.5 \pm 0.7$ & $1.0 \pm 0.5$ \\
\hline 00031030076 & 57600.7122 & 2.6 & $1.1 \pm 0.1$ & $1.7 \pm 0.2$ & $2.3 \pm 0.6$ & $1.9 \pm 0.4$ \\
\hline 00031030077 & 57604.6330 & 3.7 & $0.83_{-0.07}^{+0.08}$ & $1.8 \pm 0.2$ & $2.1 \pm 0.6$ & $1.9 \pm 0.4$ \\
\hline
\end{tabular}

Notes. ${ }^{(a)}$ Unabsorbed flux in the $0.5-10 \mathrm{keV}$ energy range derived from the model with a fixed absorption value. ${ }^{(b)}$ Photon index. 
Table A.1. Swift/XRT observations of GRO J1008-57.

\begin{tabular}{|c|c|c|c|c|c|c|}
\hline \multirow[t]{2}{*}{ Obs Id } & \multirow{2}{*}{$\begin{array}{l}\text { Date } \\
\text { MJD }\end{array}$} & \multirow{2}{*}{$\begin{array}{c}\text { Exposure } \\
\text { ks }\end{array}$} & \multicolumn{2}{|c|}{$N_{\mathrm{H}}=2.0 \times 10^{22} \mathrm{~cm}^{-2}$} & \multicolumn{2}{|c|}{ Free $N_{\mathrm{H}}$} \\
\hline & & & $\begin{array}{c}\text { XRT flux } \\
10^{-11} \mathrm{erg} \mathrm{s}^{-1} \mathrm{~cm}^{-2}\end{array}$ & $\Gamma^{b}$ & $\begin{array}{c}N_{\mathrm{H}} \\
10^{22} \mathrm{~cm}^{-2}\end{array}$ & $\Gamma^{b}$ \\
\hline 00031030078 & 57608.0913 & 3.3 & $0.81_{-0.07}^{+0.08}$ & $1.7 \pm 0.2$ & $2.6 \pm 0.7$ & $2.0 \pm 0.4$ \\
\hline 00031030079 & 57612.0746 & 3.4 & $0.93_{-0.08}^{+0.07}$ & $1.2 \pm 0.2$ & $1.2 \pm 0.4$ & $0.8 \pm 0.3$ \\
\hline 00031030080 & 57616.2037 & 3.1 & $1.1 \pm 0.1$ & $1.9 \pm 0.2$ & $3.0 \pm 0.6$ & $2.4 \pm 0.4$ \\
\hline 00031030081 & 57626.1855 & 1.4 & $0.7_{-0.1}^{+0.2}$ & $2.2 \pm 0.4$ & $0.7 \pm 0.7$ & $1.1 \pm 0.7$ \\
\hline 00031030082 & 57629.0364 & 1.7 & $1.1 \pm 0.1$ & $1.6 \pm 0.2$ & $1.1 \pm 0.5$ & $1.0 \pm 0.4$ \\
\hline 00031030083 & 57630.1009 & 1.1 & $0.9 \pm 0.1$ & $1.8 \pm 0.3$ & $2.1 \pm 0.9$ & $1.9 \pm 0.7$ \\
\hline 00031030084 & 57631.6256 & 2.1 & $1.4 \pm 0.1$ & $1.7 \pm 0.2$ & $1.3 \pm 0.4$ & $1.3 \pm 0.3$ \\
\hline 00031030085 & 57632.0994 & 2.8 & $0.98_{-0.09}^{+0.07}$ & $1.5 \pm 0.2$ & $1.5 \pm 0.5$ & $1.1 \pm 0.3$ \\
\hline 00031030086 & 57636.4050 & 0.8 & $3.0_{-0.3}^{-0.09}$ & $1.4 \pm 0.2$ & $1.8 \pm 0.5$ & $1.3 \pm 0.4$ \\
\hline 00031030087 & 57640.3242 & 3.2 & $8.7_{-0.2}^{+0.4}$ & $1.3 \pm 0.1$ & $2.6 \pm 0.3$ & $1.6 \pm 0.2$ \\
\hline 00031030088 & 57644.0456 & 3.4 & $14.1_{-0.4}^{+0.5}$ & $1.2 \pm 0.1$ & $2.0 \pm 0.2$ & $1.2 \pm 0.1$ \\
\hline 00031030089 & 57648.6950 & 3.6 & $78 \pm 2$ & $1.0 \pm 0.1$ & $2.0 \pm 0.2$ & $0.9 \pm 0.1$ \\
\hline 00031030090 & 57652.7537 & 3.3 & $159 \pm 3$ & $1.2 \pm 0.1$ & $1.5 \pm 0.1$ & $1.0 \pm 0.1$ \\
\hline 00031030091 & 57656.0031 & 3.3 & $308_{-5}^{+4}$ & $1.1 \pm 0.1$ & $1.3 \pm 0.1$ & $0.7 \pm 0.1$ \\
\hline 00031030092 & 57660.1913 & 3.5 & $360_{-5}^{+6}$ & $1.2 \pm 0.1$ & $1.2 \pm 0.1$ & $0.7 \pm 0.1$ \\
\hline 00031030093 & 57664.1124 & 3.1 & $219 \pm 4$ & $1.0 \pm 0.1$ & $1.3 \pm 0.1$ & $0.7 \pm 0.1$ \\
\hline 00031030094 & 57668.5570 & 3.4 & $54.9 \pm 1.5$ & $1.1 \pm 0.1$ & $1.8 \pm 0.2$ & $0.9 \pm 0.1$ \\
\hline 00031030095 & 57672.0878 & 2.7 & $19.1_{-0.7}^{+0.6}$ & $1.1 \pm 0.1$ & $2.0 \pm 0.2$ & $1.1 \pm 0.1$ \\
\hline 00031030096 & 57676.0145 & 1.2 & $9.1_{-0.6}^{+0.1}$ & $1.2 \pm 0.1$ & $1.9 \pm 0.4$ & $1.1 \pm 0.2$ \\
\hline 00031030097 & 57678.0084 & 1.6 & $7.1 \pm 0.1$ & $1.5 \pm 0.1$ & $2.4 \pm 0.4$ & $1.7 \pm 0.2$ \\
\hline & & & Averaged points & & & \\
\hline 00031030040-56 & - & 29.7 & $1.74 \pm 0.04$ & $1.74 \pm 0.06$ & $2.06 \pm 0.08$ & $1.75 \pm 0.09$ \\
\hline 00031030058-64 & - & 15.6 & $1.15 \pm 0.05$ & $1.84 \pm 0.07$ & $2.0 \pm 0.2$ & $1.8 \pm 0.2$ \\
\hline 00031030066-85 & - & 42.2 & $0.95 \pm 0.03$ & $1.63 \pm 0.05$ & $1.9 \pm 0.2$ & $1.5 \pm 0.1$ \\
\hline
\end{tabular}

